\title{
Integrando as Plataformas App Inventor e Arduino na Construção de um Humanoide
}

\author{
Jadson C. de Amorim ${ }^{1,2}$, Neiton Carvalho da Silva ${ }^{2}$, Marizete Silva Santos ${ }^{2}$, \\ Francisco Luiz da Silva ${ }^{1,2}$
}

\footnotetext{
${ }^{1}$ Secretaria de Educação - Secretaria Executiva de Tecnologia na Educação - Prefeitura do Recife - Av. Oliveira Lima, 824 - Soledade - Recife - PE - Brasil

${ }^{2}$ Universidade Federal Rural de Pernambuco - EADTEC(UFRPE) - Rua Dom Manoel de Medeiros, s/n, Dois Irmãos - CEP: 52171-900 - Recife, PE - Brasil

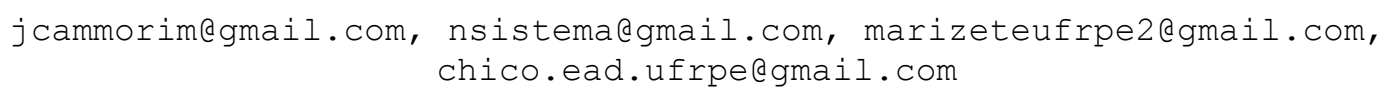

\begin{abstract}
The evolution of gadgets, $3 D$ printers, and programming softwares per blocks are transforming the Educational Robotics environment. This study presents a new reality in the construction of a Humanoid with these new technologies, enabling the elementary school students become authors in modeling, designing and programming using hardware and free software architectures. Working with prototyping platform Arduino and MIT App Inventor2, students produce not only humanoid, but applications through gadgets and Bluetooth technology control it remotely.
\end{abstract}

Resumo: A evolução dos gadgets, das impressoras 3D, e softwares de programação por blocos estão transformando os ambientes da Robótica Educacional. Este estudo apresenta uma nova realidade na construção de um Humanoide com estas novas tecnologias, possibilitando a estudantes do ensino fundamental tornarem-se autores em modelagem, construção e programação usando arquiteturas de hardware e software livre. Trabalhando com as plataformas de prototipação Arduino e MIT App Inventor2, os estudantes produzem não só o humanoide, mas aplicativos que por meio de gadgets e tecnologia Bluetooth o controlam à distância.

\section{Introdução}

Até pouco tempo a construção de artefatos robóticos nos ambientes de aprendizagem com Robótica Educacional, se limitavam a kits pré-fabricados ou a reutilização de instrumentos eletroeletrônicos em obsolescência funcional. Com o aparecimento das impressoras 3D, e o uso popular de gadgets inteligentes, esta realidade está sofrendo modificações, este espaço de aprendizagem se transforma em ambiente de produção autoral com estudantes e professores envolvidos na construção de artefatos cada vez mais originais e complexamente modelados como a exemplo de um humanoide.

A construção de um humanoide, e a viabilidade em desconstruí-lo e reconstruí-lo em atividades pedagógicas, aproximam os jovens aprendizes a uma tecnologia de ponta por muitos ainda desconhecida, trazendo o futuro para o presente e as imagens virtuais para a prática. Essa transformação paradigmática, do aprender diante do novo, da ação em 
desenvolver novas habilidades, é evidenciado por Papert (1994, p. 5) quando coloca que "...a habilidade competitiva será a habilidade de aprender."

A automação e controle de um artefato robótico consiste em parte complementar e final na sua construção, considerada até pouco tempo como etapa mais complexa desse processo e acessível apenas na graduação. Diante da evolução dos sistemas operacionais, recursos e novas linguagens de programação para os atuais smartphones e tablets, esta fase de construção de robôs tem se tornado realidade para salas de aula do ensino fundamental.

O crescente aumento na aquisição destes gadgets por diferentes classes sociais segundo a consultoria IDC Brasil (2015) o trouxe também para os espaços da escola. Esta inserção, alinhada ao crescimento no acesso a internet e ao surgimento da computação nas nuvens, ampliaram os espaços de discussão e investigação de uso dos gadgets nos processos educacionais formais e não formais, propiciando com estes instrumentos o aumento da procura por aplicativos educativos e seus ambientes de produção.

Ainda de acordo com a consultoria da IDC Brasil, em janeiro 2015 o uso de smartphones no Brasil chegou a 70,3 milhões de usuários, sendo colocado em $6^{\circ}$ lugar em número de aparelhos e usuários, abaixo apenas da China, USA, Índia, Japão e Rússia. Quatro destes países, encontram-se em plena ascensão na produção de robôs humanoides, com investimentos expressivos e intercâmbio entre empresas e universidades, como o ICub, as criaturas Poppy, o Asimo e outros. Diante destes fatos e uso destas e outras plataformas já é possível a produção e automação de diferentes robôs nas escolas de educação fundamental, dentre eles os humanoides.

Com a disponibilidade desses recursos tecnológicos uma nova realidade é descortinada para o ensino fundamental, quando as discussões sobre tal importância é retomada por pesquisadores e educadores brasileiros sendo percebida por outros países, sobre o ensino de computação para crianças e adolescentes, envolvendo o ensino de linguagem de programação e mais amplamente do pensamento computacional no desenvolvimento de competências e habilidades que de fato contribuem na transformação de estudantes usuários em desenvolvedores de soluções.

É preciso, então, garantir que qualquer atividade de aprendizagem ocorra de modo significativo, prazeroso e realizada a partir da construção de aplicações que tenham utilidades práticas no mundo real. Desta forma, este trabalho apresenta uma experiência de construção de um humanoide com a participação de estudantes do ensino fundamental, envolvidos desde a idealização das características externas do humanoide, à escolha de sua arquitetura de hardware e software de comando.

O robô, como recurso, é um desencadeador de motivação intrínseca e aprendizagens significativas, passando a fazer parte de um grande processo de múltiplas linguagens voltadas para o desenvolvimento da autonomia de construção do conhecimento por estudantes em nível de ensino fundamental. 
V Congresso Brasileiro de Informática na Educação (CBIE 2016)

Anais do XXII Workshop de Informática na Escola (WIE 2016)

\section{Metodologia no Projeto Humanoide}

Para viabilizar qualquer processo pedagógico é indispensável um bom planejamento das etapas a executar, ter conhecimento sobre o público alvo e utilizar recursos didáticos que os motive a desenvolver as atividades com prazer e dedicação.

Tendo como objetivos a construção, programação e controle de um humanoide voltado para atividades pedagógicas, este trabalho descreve não só o processo com suas etapas e recursos, mas também uma análise de sua importância para construção de competências e habilidades pelos estudantes envolvidos diante das novas tecnologias. Através da realização de atividades presenciais e a distância, o experimento foi conduzido por pesquisas sobre humanoides, modelagem da estrutura corporal do robô, ensaios de programação para movimento de motores, construção de algoritmos em código e blocos, buscando principalmente o desenvolvimento da autonomia na construção da aprendizagem.

As etapas citadas inserem os princípios do pensamento computacional indispensável nas interações diárias com as novas tecnologias e na construção de artefatos robóticos. França (2015) coloca-nos que a Computação enquanto saber necessário na educação básica, engloba princípios fundamentais como a teoria da computação, e incorpora técnicas e métodos, como a abstração e raciocínio lógico, o que deve ser considerado, pois podem ser aplicados na solução de problemas e no avanço do conhecimento.

A integração dos processos pedagógicos, as tecnologias, e os princípios da computação, tendo a robótica como instrumento motivador pode oferecer de fato, segundo ZILLI (2004 p.14), um ambiente de aprendizagem que prioriza a forma de aprender de cada indivíduo na sua diversidade, oferecendo múltiplos estímulos como a visão, a audição e o tato simultaneamente.

As atividades com o uso do App Inventor, na construção de aplicativos para a comunicação com o Arduino e controle das ações e reações do humanoide, foram precedidas por experimentos utilizando o ambiente Code e o aplicativo Scratch. Essas duas plataformas de programação, estruturadas na programação por ícones ou blocos, facilitam o processo de aprendizagem para o desenvolvimento da lógica de programação e construção de algoritmos.

Diante das habilidades trabalhadas com o desenvolvimento do pensamento computacional, foi iniciada a produção do aplicativo de controle do humanoide para gadgets com o sistema operacional Androide. O ambiente virtual do App Inventor foi escolhido para esta produção devido as facilidades de entendimento por parte dos estudantes, assim como o ambiente do Arduino como plataforma de prototipação do projeto, desenvolvendo paralelamente as etapas de impressão, montagem, adaptação e remontagem do robô.

Neste período, outros programas foram utilizados para testar individualmente cada um dos motores do robô, primeiro por meio do envio de comandos via porta serial para o Arduino; em seguida utilizando diferentes tecnologias embarcadas nos smartphones como sensores de equilíbrio e posicionamento GPS, finalizando com programas simples para controle de robôs com rodas. 
Participaram do projeto 43 estudantes de escolas públicas do ensino fundamental de $6^{\circ}$ ao $9^{\circ}$ ano, com faixa etária entre 10 e 14 anos, envolvidos e motivados com o Programa Robótica na Escola. Com o referido programa cada unidade escolar recebeu diversos kits de robótica com material LEGO. A participação e interação no modelo à distância também foi implementado, utilizando para tal um ambiente AVA abordando conteúdos de Robótica Educacional. Presencialmente, foram desenvolvidas atividades integrando a robótica com os professores e as disciplinas de Matemática, Ciências, Geografia e Eletiva de Robótica.

Concomitantemente, de forma voluntária e autônoma, doze dos quarenta e três estudantes envolvidos no projeto, frequentaram o laboratório do Programa de Robótica da Secretaria de Educação do Recife, onde além da construção do Humanoide desenvolveram outros projetos robóticos de interesse individual e coletivo. Este laboratório está estruturado em três espaços físicos e linhas de robótica específicas, mas que se integram no fazer pedagógico

Um dos laboratórios é representado pela Robótica com Ferramentas ou Robótica Livre, estruturando o uso de elementos oriundos da reutilização de instrumentos eletroeletrônicos em obsolescência. A Robótica de Encaixe com material LEGO está instalado no segundo laboratório, desenvolvendo atividades com kits para cada nível educacional, no terceiro está a Robótica Avançada com trinta Humanoides NAO desenvolvendo desde projetos em parceria com universidades ao atendimento a estudantes com Autismo em sala de aula.

Grande parte deste projeto foi realizada no laboratório da Robótica com Ferramentas, espaço com instrumentos e materiais característicos para a construção de artefatos originais. Entre os trabalhos desenvolvidos com o humanoide, os estudantes do projeto eram convidados a exercer a função de monitor em atividades de iniciação com outros estudantes, exercitando assim o desenvolvimento da habilidade do aprender ao ensinar, possibilitando a observação de sua evolução cognitiva e habilidade como mediador de aprendizagens.

\section{O Humanoide}

Para projetar o humanoide, foi utilizada a tecnologia de impressão 3D, na expectativa de sua popularização e consequente barateamento, tornando o projeto de baixo custo, tecnicamente reproduzível, permitindo a estudantes e professores replicar, além de todo o projeto, novas peças de forma própria e personalizada. Desmistificando assim, a remoção das restrições de fabricação de produções técnicas clássicas como um humanoid, mostrado nas imagens a seguir. 


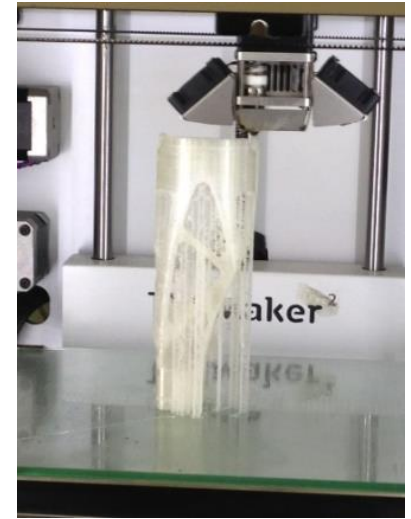

Figura 1. Impressão

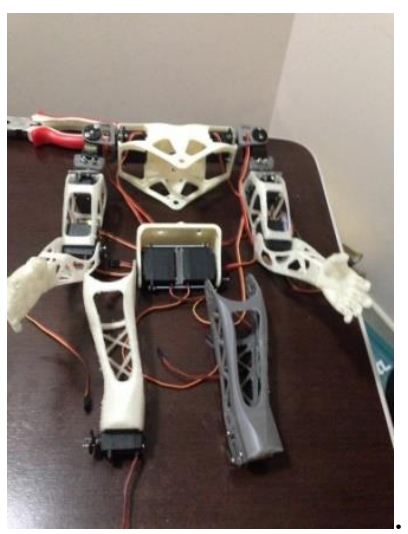

Figura 3. Corpo

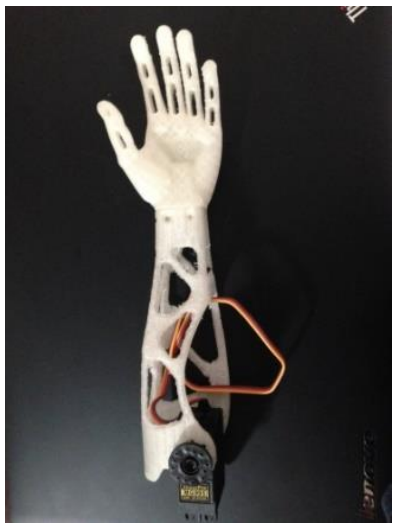

Figura 2. Antebraço e mão

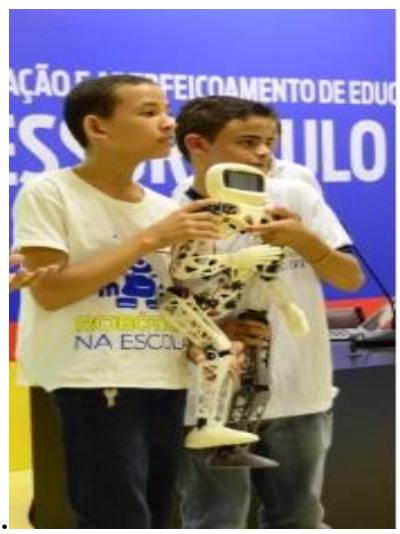

Figura 4. Estudantes e robô

\section{O App Inventor 2}

Este ambiente de programação de interface visual bastante intuitiva, permite às pessoas que desconhecem as linhas de código de programação, que seus próprios projetos sejam construídos com relativa facilidade. O desenvolvimento de aplicativos é permitido por sua estrutura direto nas "nuvens", podendo ser baixados e executados nos referidos gadgets, ou no emulador disponibilizado no próprio ambiente.

A interface do ambiente está dividida em duas partes: o ambiente de Designer e o ambiente Blocks Editor. No ambiente Designer constroi-se o projeto no formato visual, inserindo botões, imagens, textos, enquanto no ambiente Blocks Editor preparase o algoritmo de programação em forma de blocos, imagens que se encaixam quando arrastadas e soltas próximas às outras, requisitando apenas algumas habilidades de racioncínio lógico, um bom planejamento do que se quer produzir, e o interesse em pesquisar.

\subsection{Ambiente do MIT App Inventor 2}

A interface do ambiente de programação do App Inventor 2, apresenta o design mostrado na Figuta 5, identificado suas partes sequencialmente enumeradas. 
V Congresso Brasileiro de Informática na Educação (CBIE 2016)

Anais do XXII Workshop de Informática na Escola (WIE 2016)

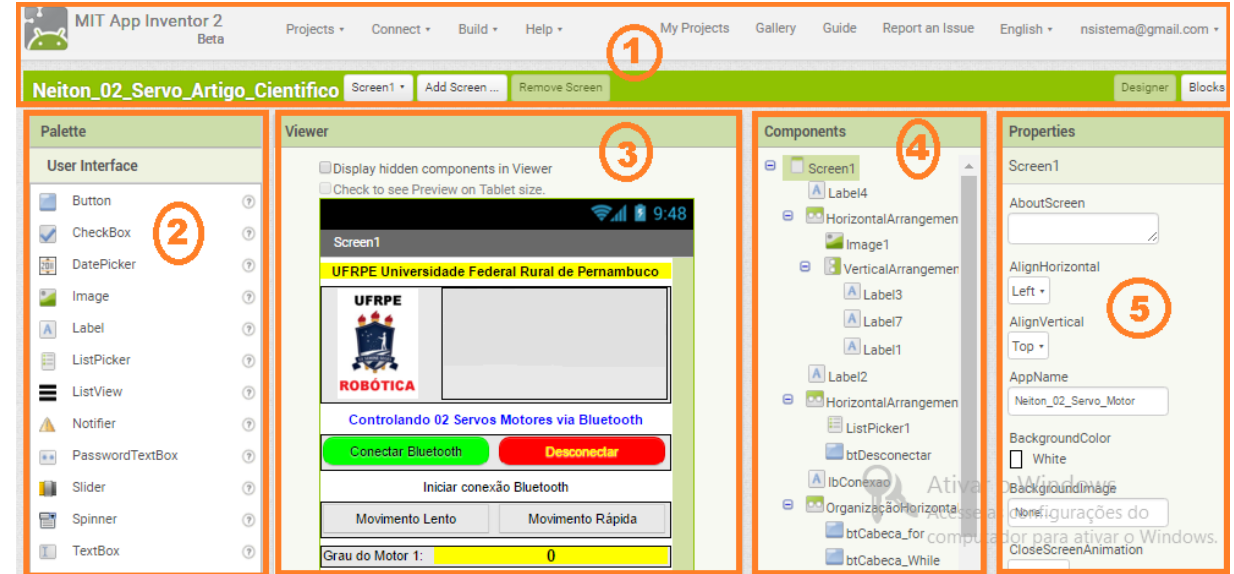

Figura 5. Interface do MIT App Inventor 2

Área 1- Barra de menu

Área 2 - Barra de paleta

Área 3 - Tela do App

Área 4 - Paleta deomponents

Área 5 - Paleta de propriedades

Utilizando o ambiente descrito acima foi produzido a o App planejado para o humanoide, o qual apresenta, em uma de suas interfaces, elementos como os programados e identificados na Figura.6, com seus respectivos nomes e funções.

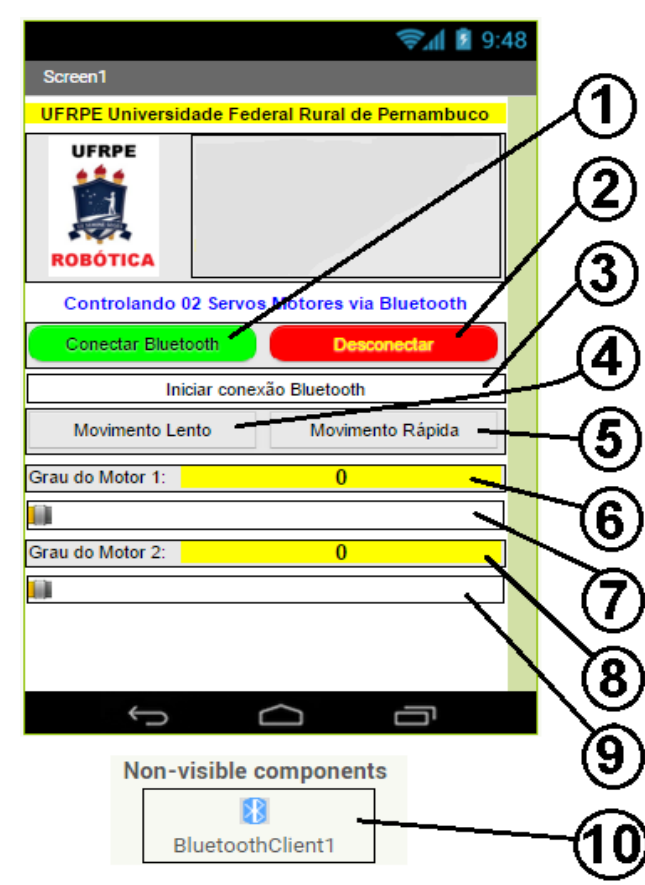

1-ListPicker1 - botão que realiza a varredura dos dispositivos de bluetooth disponíveis para conexão;

2-btDesconectar - botão para desconectar o dispositivo pareado ou conectado;

3-lbConexao - indicação de conexão ou desconexão com o dispositivo;

4-btMovimentoLento - acionar movimentos lentos nos servos motores;

5-btMovimentoRapido - acionar movimentos rápidos nos servos;

6 e 8-1bGrauMotor1- posicionamento em graus do servos;

7 e 9-SliderServoMotor1 - botão deslizante para alteração da posição do servo-motor;

10-BluetoothClient1 - Componente oculto de

Figura 6. Elementos programados no App Inventor 2

\subsection{Programações com o App Inventor}

Na perspectiva da construção coletiva, os movimentos foram idealizados pelos estudantes para as partes do humanoide durante as atividades de modelagem, impressão e montagem utilizando os recursos do MIT App Inventor 2 representadas a seguir. Na Figura.7 a programação se destina a conexão com o modulo bluetooth, identificando-a quando positiva na cor verde. 
V Congresso Brasileiro de Informática na Educação (CBIE 2016)

Anais do XXII Workshop de Informática na Escola (WIE 2016)

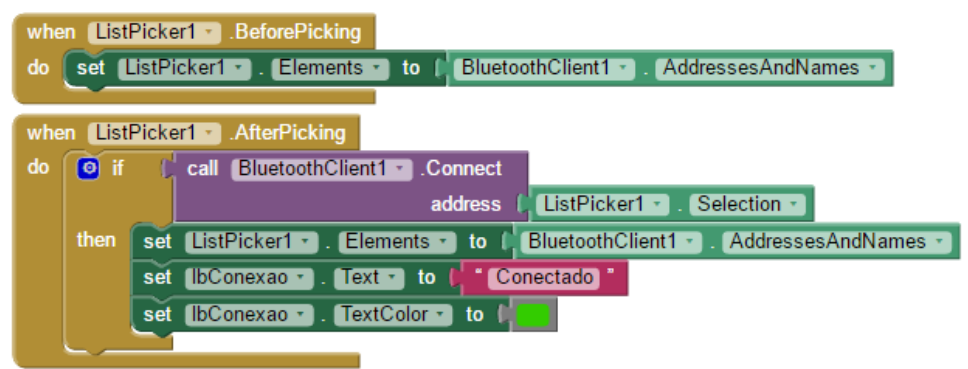

Figura 7. Conexão bluetooth

\subsection{Fluxo lógico do Processo}

A partir de um smartphone ou tablete, com sistema operacional Android, é instalado o App construído especificamente para o controle do humanoide e suas partes. O aplicativo, acionado por meio de um click, realiza o pareamento do gadget com o módulo bluetooth conectado ao Arduino, possibilitando assim a transmissão dos comandos de acionamento de sua programação com a do Arduino, onde estão os códigos de controle do estado dos servos motores.

Ao escolher um ícone de comando de uma ou mais partes do corpo do robô no aplicativo, pode-se acioná-lo por meio de um clique, ou um clique e arrasto de um slider, assim acionando um comando direcionado ao módulo Bluetooth e deste para o Arduino.

O aplicativo apenas chama a função ou comando que pretende executar, enquanto o algoritmo instalado no Arduino é que faz funcionar as instruções de acionamento ou desligamento dos pinos em que estão conectados, passando ou não por um shield, e deste para cada servo motor, sensor, módulo de vídeo ou áudio, como está representado na Figura.8 a seguir.

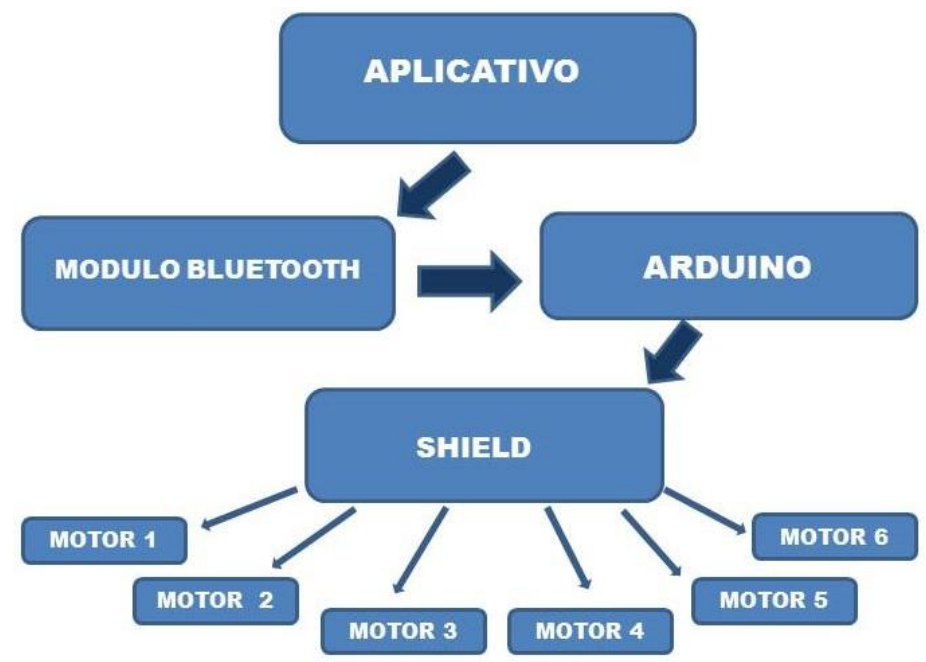

Figura.8. Fluxo da interação entre um Smartphone ou Tablet e o Humanoide

\section{A Plataforma Arduino}

O Arduino é um projeto que engloba software e hardware e tem como objetivo fornecer uma plataforma de fácil manipulação para prototipação de projetos interativos, 
V Congresso Brasileiro de Informática na Educação (CBIE 2016)

Anais do XXII Workshop de Informática na Escola (WIE 2016)

utilizando um microcontrolador. Ele faz parte do que é chamado de computação física: area da computação em que o software interage diretamente com o hardware JUSTEN (2009 p.8)

\subsection{Algoritmo do Arduino para os servos motores}

A construção da programação no Arduino, foi evoluindo a partir da experimentação de códigos exemplos, utilizados em protótipos móveis, construídos com material de eletroeletrônicos em obsolescência. Estes códigos aqui apresentados, são o princípio do trabalho, pois sua construção não tem fim diante das atividades pedagógicas a serem implementadas com o humanoide, quando o mesmo estiver em uso na sala de aula.

Nesta perspectiva, os desafios estarão sempre motivando os estudantes a pesquisar, construir, expor e testar suas hipóteses com colegas e professores. A imagem da Figura.9, representa uma das partes do código original criado para inserir o controle dos servo motores para execução das primeiras ações do robô.

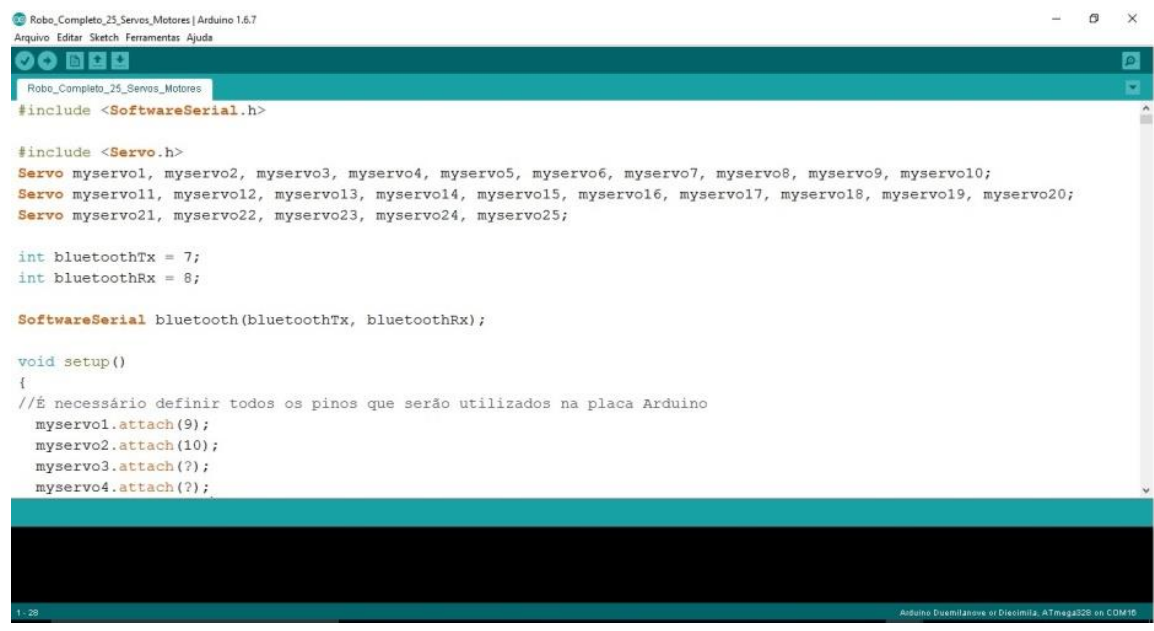

Figura 9. Sketch com código inicial do robô

Estes, a construção do algoritmo e a produção do sketch na IDE do Arduino é um dos desafios em que os estudantes necessitam de um reforço na motivação, resultado positivo apresentado na transcrição da fala de um dos estudantes participantes do projeto.

“...primeiro porque foi uma ótima experiência construir um humanoide e sendo ele feito por mim e os meus colegas, foi ainda mais feliz. Aprendemos a programar isso foi muito legal, divertido ao longo da construção, aprendi o quanto é importante estudar em sala de aula e discutir com o professor quando não entendemos,..."

\subsection{Circuito de instalação dos componentes}

Com o desenvolvimento de habilidades para entender e representar um circuito elétrico básico, diferentes softwares foram utilizados pelos estudantes on-line e no computador. A partir desta aprendizagem, representaram o circuito de comunicação e controle dos 
V Congresso Brasileiro de Informática na Educação (CBIE 2016)

Anais do XXII Workshop de Informática na Escola (WIE 2016)

motores com o Arduino e o modulo Bluetooth utilizando o Fritzing, uma aplicação Open Source e de fácil operação na simulação de projetos como mostrado na Figura.10.

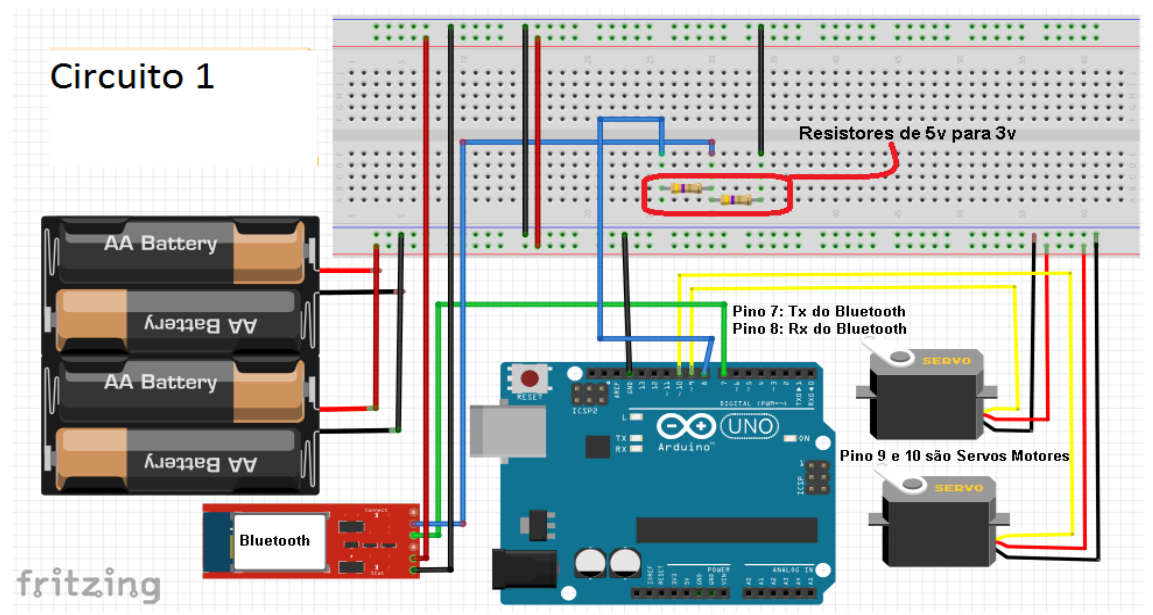

Figura 10. Circuito com Arduino, servos motores e módulo Bluetooth

\section{Considerações Finais}

Os resultados apresentados nesta experimentação, de fato contribuem para demonstrar o desenvolvimento de habilidades voltadas para a construção de algoritmos e programação com estudantes de ensino fundamental, assim como o desenvolvimento de aprendizagens necessárias como pré requisitos na construção de aplicativos produzidos através de linguagens de programação com criatividade e funcionalidade. Também fica evidenciado que a linguagem de código utilizada para Arduino facilita a construção de comandos para a automação dos movimentos planejados para um robô.

Concluímos que no desenvolvimento de atividades com pensamento computacional se faz necessário o uso de estratégias que envolvam a resolução de problemas e que promovam desafios individuais de forma atrativa. Nesta perspectiva, é possível ensinar conceitos complexos com métodos de aprendizagem dinâmicos e lúdicos, implementando um espaço multirreferencial com recursos motivacionais e analogias que despertem o interesse dos estudantes facilitando o aprendizado dos conceitos de programação.

O App Inventor integrado ao uso de experimentos com a plataforma Arduino, apresenta-se, dentro da metodologia utilizada, como recurso eficaz para o desenvolvimento da abstração, raciocínio lógico e resolução de problemas por meio da programação.

\section{Referências}

Arduino. Site Oficial disponível em https://www.arduino.cc. Acesso em 10 de fevereiro de 2016.

Bell, Tim. Witten, Ian H. Fellows, Mike. CS Unplugged - An enrichment and extension programme for primary-aged students. 2011.

César, Danilo Rodrigues. Robótica pedagógica livre: uma alternativa metodológica para a emancipação sociodigital e a democratização do conhecimento - 2013. 
V Congresso Brasileiro de Informática na Educação (CBIE 2016)

Anais do XXII Workshop de Informática na Escola (WIE 2016)

Dansk Arduino Forum - Forum. Disponível em: <http://arduino-forum.dk/wpcontent/uploads/2014/03/Bluetooth_modem_arduinokode.pdf $>$

Evans, Martin. Joshua Noble, Jordan Hochenbaum. Arduino em Ação. Novatec Editora. São Paulo 2013.

Finizola, Antônio Braz at al. O Ensino de programação para dispositivos móveis utilizando o MIT-App Inventor com alunos do ensino médio. 2014.

França, Rozelma S., Patrícia Tedesco. Explorando o pensamento computacional no ensino médio: do design à avaliação de jogos digitais. WIE 2015.

Fritzing. Disponível em: < http://fritzing.org/download/. Acesso em 16 de abril de 2016

Github - Site GitHub Gist. Disponível em: https:/gist.github.com/. Acesso em 21 de março de 2016.

ISTE; CSTA; NSF. (2011). Computational thinking: leadership toolkit. First Edition

Justen, Alvaro. Curso de Arduino. Disponível em: http://www.cursodearduino.com.br/apostila. Acesso em 11 de fevereiro de 2016

McRoberts, Michael. Arduino Básico. Novatec Editora, São Paulo, 2011.

Mit APP Inventor - APP Inventor. Disponível em: http://appinventor.mit.edu/ explore/http://veja.abril.com.br/noticia/economia/venda-de-smartphones-sobe-55-nobrasil-em-2014. Acessado em 01/05/2016.

Monk, Simon. Projetos com Arduino e Android: use seu smartphone ou tablet para constrolar o Arduino. Porto Alegre. Bookman 2014.

Servo Library - Site Oficial Arduino. Disponível em: file://C:/Program\%20Files/Ard uino/reference/arduino.cc/en/Reference/Servo.html

Sousa, R. V. et al. (2010). "Ensinando e aprendendo conceitos sobre a ciência da computação sem o uso do computador: Computação Unplugged!”. Práticas em Informática na Educação: Minicursos do CBIE, v.1, n.1.

Wing, Jeannette M. Computational thinking. Communications of the ACM, v. 49, n. 3, p. 33-35, 2006.

Zilli, Silvana do Roccio. A Robótica Educacional no Ensino Fundamental: Perspectivas e Prátic. Florianópolis, 2004. 